Bulletin de la Société Royale des Sciences de Liège, Vol. 85, 2016, p. 1204 - 1210

\title{
Improving BCI performance using SSA and GA
}

\author{
Mohammad SoURI*, Sassan AZADI ${ }^{\dagger}$ \\ Department of Biomedical Engineering, Semnan University, Semnan, Iran
}

\begin{abstract}
Brain Computer Interface (BCI) systems based on motor imagery tasks have significant usage for disabled people for their life style. Electroencephalogram (EEG) is one of the best approach to obtain human brain signals. The EEG signal requires three stages of preprocessing, feature extraction, and classification to increase signal analysis accuracy. Due to different task of brain, EEG distribution fluctuates (non-stationary), and therefore challenges BCI for many researchers. Recently a method named stationary subspace analysis (SSA) applied to some BCI data by some research teams in order to separate stationary and non-stationary parts of EEG. However they did not obtain significantly results. This method factorizes EEG into its stationary and non-stationary components by dividing signal into number of epochs, and compares their data distributions. In this study, we applied SSA in preprocessing stage into train and test data of the BCI competition dataset of nine healthy people. We applied the Genetic Algorithm (GA) to train our Artificial Neural Network (ANN) classifier. We also inspect different parameters for SSA to improve the performance. Our results indicate significant growth especially for subjects with worse results in other techniques (improving $40 \%$ to $\sim 70 \%$ ). In addition, the mean of accuracies improves $5 \%$ in regard to the winner of competition.
\end{abstract}

Keywords: BCI, motor imagery, Artificial Neural Network, Genetic Algorithm, Stationary Subspace Analysis, Feature extraction, Classification

\section{Introduction}

A brain computer interface (BCI) is an intellect system which can distinguish a certain set of template in brain signals. In addition it referred to a brain machine interface (BMI) which developed to aid disabled people for performing their daily activities. One challenge issue for systems of BCI is motor imagery (MI) tasks based electroencephalography (EEG) signals [1]. BCI consist of a hardware and software communications system that provides a condition for humans to interact with their environments in while circumferential nerves and muscles have not any involvement. In the last decades, development of BCI has received attention for being a valuable new additive augmentative communication option for disabilities. The most applicable of BCI solution are based on electroencephalography (EEG) measurements of scalp. EEG, an

\footnotetext{
${ }^{\dagger}$ Email address : $\underline{\text { sazadi@ @emnan.ac.ir }}$
} 
Bulletin de la Société Royale des Sciences de Liège, Vol. 85, 2016, p. 1204 - 1210

activity of functional brain related to change of surface potentials on the surface of scalp, uses various methods for detecting brain activities such as electrode placements, filtered and processed [2].

To predict and identify, we need to classification techniques. Feature extraction in BIC is an important task. A great classification rate could be obtained if extracted features are efficient for differentiating MI tasks. So for having a suitable classifier, it needs to be designed. There are several methods of feature extraction for MI data which applied in applications of BCI such as fast Fourier transform (FFT) [3] and wavelet coefficients [4].

The use of EEG signals for connection between humans and computers has been possible by computer hardware and signal processing. There are many methods for categorize the EGG signals [5]-[8].

For example, neural network (NN) [8], Linear discriminant analysis (LDA) [6] and Support Vector Machine (SVM). Sun et al. presented a method of on-line classification for BCI based on common Spatial Pattern (CSP) for feature extraction and SVM as a classifier. They published the best classification result for three subjects of \% 86.3, \%91.8 and \% 92 [9].

Two different neural networks and support vector machine is used to classify features which they are extracted by wavelet transform by M. R. Nazari Kousarrizi and his teammates [10].

The cross correlation based logistic regression (CC-LR) algorithm is shown as a classification of motor imagery tasks for BCI applications by Siuly [1]. They studied an insight into how to select a reference channel for cross correlation (CC) technique and applies three diverse feature sets separately, as the input to the logistic regression (LR) classifier. In 2013, Kottaimalai R along with his colleague investigates an Artificial Neural Network (ANN) in order to classify the mental tasks, which signals of brain are trained using neural network and principal component analysis (PCA) with ANN. "By PCA and neural network, the redundant data is omitted in dataset and obtained data is trained by using neural network $(\mathrm{NN})$. It is shown the correctly classified percentage of data is better in PCA with $\mathrm{NN}$ to compare to $\mathrm{NN}$ alone [11].

\section{Problem Statement}

\section{Dataset}

The selected dataset for this paper is IIa from BCI competition IV which have provided by the Department of Medical Informatics, Institute for Biomedical Engineering, University of Technology Graz. They recorded EEG signal by 22 electrodes from 9 healthy people and also EOG signal in order to provide possibility of estimation of EOG influence by 3 electrodes. The cue-based BCI paradigm consisted of four different motor imagery tasks, namely the imagination of movement of the left hand (class 1), right hand (class 2), both feet (class 3), and tongue (class 4) [12]. $\mathrm{Ag} / \mathrm{AgCl}$ mono-polar electrodes have been used with $250 \mathrm{~Hz}$ sampling rate.

Recording have been performed in 2 sessions on 2 different days for each subject and each session embraces six runs that they separated by a short breaks. There are 12 trials for each class in each run which makes 288 recorded trials in each session. They passed the signals of each channels through a filter of $[0.5,100] \mathrm{Hz}$ and also another notch filter of $50 \mathrm{~Hz}$ to conquer line noise.

During the test, subjects are sited in a comfortable armchair in front of a computer screen. At the beginning of a trial $(t=0.0 \mathrm{sec})$, monitor displays a fixation cross and also a short acoustic warning 


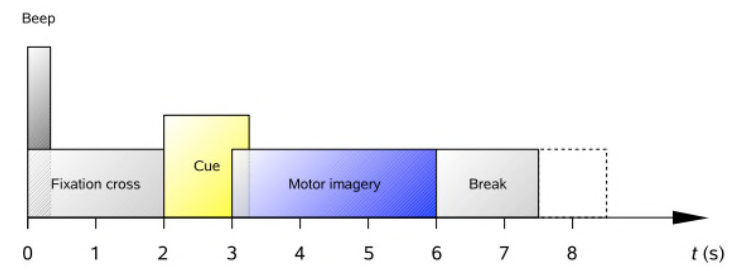

Fig1: Timing scheme of the paradigm[12]

tone plays. After two seconds $(\mathrm{t}=2 \mathrm{sec}$ ), an arrow forms cue pointing either to the left, right, down or up (representing one of the four classes left hand, right hand, foot, or tongue) appears and remains on the screen for $1.25 \mathrm{sec}$. It provokes the subjects to perform the desired motor imagery task. The subjects are trained to perform the displayed motor imagery task until the fixation cross remains on the screen at $\mathrm{t}=6 \mathrm{sec}$. Trial ends with a break and screen shows black color during this break. Figure 1 demonstrates this paradigm.

In this work, we use one session for the training and the other session for the test.

\section{Stationary Subspace Analysis}

Stationary Subspace Analysis (SSA) [13] is an innovative technique to factorize a highdimensional multivariate time-series into its stationary and non-stationary components. Its basic assumption is based on the signal $x(t)$ is a linear superposition of $d$ stationary $S_{g}$ and $D-d$ nonstationary $\mathrm{s}_{\mathrm{n}}$ sources

$$
x(t)=A S(t)=\left[\begin{array}{ll}
A_{g} & A_{n}
\end{array}\right]\left[\begin{array}{l}
S_{g}(t) \\
S_{\mathrm{n}}(\mathrm{t})
\end{array}\right]
$$

where $A$ is an invertible matrix. SSA finds a linear transformation $A^{-1}$ that distinguishes the ssources from the n-sources in a signal. In order to do this process, the $x(t)$ signal is divided into epochs and an optimization algorithm using to separate the sources. More precisely, SSA separates sources in a way which the distance measured as Divergence of Kullback-Leibler $\left(D_{K L}\right)$ between the distribution of the estimated s-sources in each epoch (described by first two moments) and the standard normal distribution is minimized [14].

With attention to this model, the s-sources and the n-space are uniquely identifiable whereas the $\mathrm{n}$-sources and the s-space are not [15].

In general it has been shown in [13] that at least of epochs number depends on number of nonstationary sources,

$$
N>\frac{D-d}{2}+2
$$

where $N$ is epochs number.

\section{Proposed methodology}

EEG signal have lots of different sources in brain which it causes to create lots of changes in EEG distribution during time. In order to strengthen the BCI against these continual changes, we employ SSA in pre-processing step to limit the classification to the stationary part of EEG. To achieve this goal in this paper, the EEG signal is divided into epochs which long as some quantity of consecutive trials in order to make sure that all classes appear in all epochs which it cause to prevent SSA to assume some different between classes as non-stationary element. 


\section{Feature Extraction}

In this study, we use Barlow parameters, Hjorth parameters, band power estimation and some of time domain parameters as extracted features. Hjorth parameters consist of the signal power named activity, mean frequency named mobility and change in frequency named complexity. Barlow parameters consist of Spectral Purity Index (SPI, used to measure the irregularity of the signal), Amplitude (A) and Frequency (F). Log (BP) is commonly used in many BCI systems [16]. The signal in each channel is band-pass filtered using Butterworth filters of order 5 at definite frequency. The logarithm is applied to all features to make them linear then they finally normalized before entering the classifier.

\section{Classification with Artificial Neural Networks Trained with Genetic algorithm}

In this work, a five-layered feed-forward $\mathrm{NN}$ is used and trained by Genetic algorithm. At first, the genetic algorithm produces a primary population which consists of 30 different populations as different weights and biases for $\mathrm{NN}$ and sort them by the result accuracy in $\mathrm{NN}$, then next generation is produced by choose and mix best parents and also some random populations and sort them again. This process repeated for 25 generation, then the most accurate population finalize in the $\mathrm{NN}$ as its biases and weights.

\section{Results and Discussions}

The classification accuracy is the most generally used criterion to value the efficiently of different techniques in pattern recognition problems. The accuracy of a measurement system is the degree of closeness of measurements of a quantity to its actual (true) value [1]. The classification accuracy calculated by a fraction which has the number of correctly classified trials as numerator and the total number of trials as denominator.

Figure 2 shows the effect of different estimation of number of stationary sources, $d$, for the subject number 1 .

According to many runs with different initial value in this work, choosing 16 ford gives us the best accuracy. Figure 3 compares the accuracy of this technique with different numbers of trials per epoch, what is important is the fact that this parameter must be divisible to total numbers of trials in each session (288). With attention to equation 2 and choosing $d=16$, the number of trials per epoch must be less than 50 in order to have at least 5 epochs.

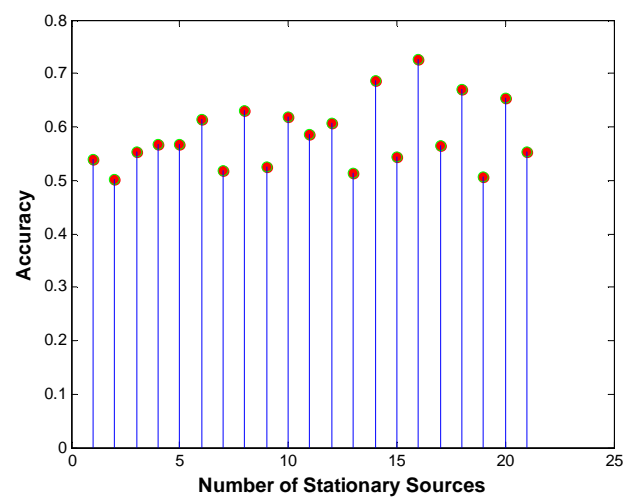

Fig.2: Effect of different number of stationary sources on accuracy 


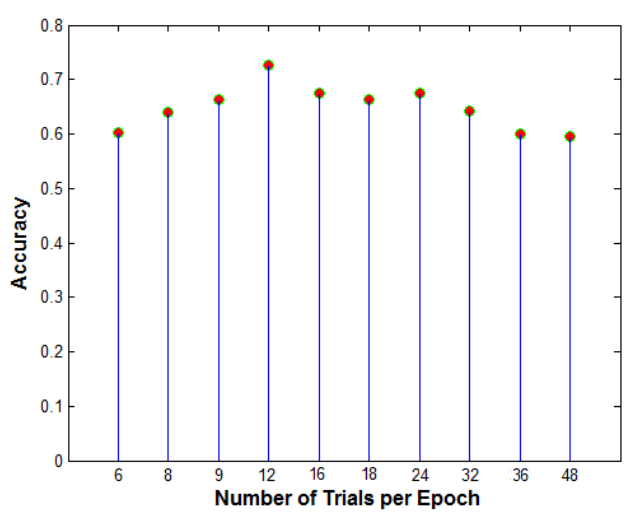

Fig. 3:Effect of choosing different number of trials per epoch

Choosing 12 trials in each epoch have the best result between other numbers.

Table 1 compares the result against not only the winner of the competition which held by Department of Medical Informatics, Institute for Biomedical Engineering, University of Technology Graz [17] on the suggested data set but also back propagation ANN (shown by ANN in table 1). The results show that GA ANN presents better results in comparison with other techniques.

Table 1: The accuracy of using SSA with GA trained ANN and ANN without GA comparing winner of the competition for all nine subjects

$\begin{array}{ccccccccccc} & \text { S1 } & \text { S2 } & \text { S3 } & \text { S4 } & \text { S5 } & \text { S6 } & \text { S7 } & \text { S8 } & \text { S9 } & \text { Mean } \\ \text { GA ANN } & 0.64 & 0.50 & 0.70 & 0.61 & 0.46 & 0.60 & 0.74 & 0.75 & 0.57 & 0.61 \\ \text { ANN } & 0.52 & 0.53 & 0.52 & 0.52 & 0.48 & 0.59 & 0.61 & 0.55 & 0.53 & 0.54 \\ \text { Winner } & 0.68 & 0.42 & 0.75 & 0.48 & 0.4 & 0.27 & 0.77 & 0.75 & 0.61 & 0.57\end{array}$

\section{Conclusion}

We investigated the using effect of SSA and employment of more effective algorithm for training ANN in this paper. Based on table 1, GA ANN showed the best results in comparison with other techniques. It gives significantly better accuracy in persons who had more noises or maybe less focus on their test such as subject 6 . However, we could see the accuracy of GA ANN decreases significantly in the subjects which have some missing part in their signals because of using SSA for instance subject 9.

Ordinary ANN classifier (Back propagation ANN) illustrates the different pattern in accuracies for subjects which it proves remarkable effect of train algorithm in neural networks. 
Bulletin de la Société Royale des Sciences de Liège, Vol. 85, 2016, p. 1204 - 1210

\section{References}

[1] Siuly, Y. Li, and P. Paul Wen, "Modified CC-LR algorithm with three diverse feature sets for motor imagery tasks classification in EEG based brain-computer interface.," Comput. Methods Programs Biomed., vol. 113, no. 3, pp. 76-80, Mar. 2014.

[2] W. Mansor and L. Y. Khuan, "A Review of Signal Processing in Brain Computer," no. December, pp. 443-449, 2010.

[3] K. Polat and S. Gunes, "Classification of epileptiform EEG using a hybrid system based on decision tree classifier and fast Fourier transform," Appl. Math. Comput., vol. 187, 2007.

[4] W. Ting, Y. Guo-Zheng, Y. Bang-Hua, and S. Hong, "EEG feature extraction based on wavelet packet decomposition for brain-computer interface," Measurement, vol. 41, pp. 618-625, 2008.

[5] E. Abbas and G. Mahdi, "EEG signals can be used to detect the voluntary hand movements by using an enhanced resource-allocating neural network," Eng. Med. Biol. Soc. Proc. IEEE Int. Conf. Ann., vol. 1, pp. 721-724, 2001.

[6] D. Garrett, A. P. David, C. W. Anderson, and M. H. Thaut, "Comparison of linear, nonlinear, and feature selection methods for EEG signal classification," IEEE Trans. Rehab. Eng., vol. 11, pp. 141-144, 2001.

[7] A. F. Cabrera, D. Farina, and K. Dremstrup, "Comparison of feature selection and classification methods for a brain-computer interface driven by non-motor imaginary," Med Biol Eng Comput, vol. 48, pp. 123-132, 2010.

[8] G. Pfurtscheller, J. Kalcher, C. Neuper, D. Flotzinger, and M. Pregenzer, "On-line EEG classification during externally-placed hand movements using a neural network-based classifier," Electroen. Clin. Neurophysiol, vol. 99, pp. 416-425, 1996.

[9] H. Sun, Y. Xiang, Y. Sun, H. Zhu, and J. Zeng, "On-line EEG classification for braincomputer interface based on CSP and SVM," 2010 3rd Int. Congr. Image Signal Process., pp. 4105-4108, Oct. 2010.

[10] M. R. N. Kousarrizi, A. A. Ghanbari, M. Teshnehlab, M. A. Shorehdeli, and A. Gharaviri, "Feature Extraction and Classification of EEG Signals Using Wavelet Transform, SVM and Artificial Neural Networks for Brain Computer Interfaces," 2009 Int. Jt. Conf. Bioinformatics, Syst. Biol. Intell. Comput., pp. 352-355, 2009.

[11] R. Kottaimalai, M. P. Rajasekaran, V. Selvam, and B. Kannapiran, "EEG signal classification using Principal Component Analysis with Neural Network in Brain Computer Interface applications," 2013 IEEE Int. Conf. Emerg. Trends Comput. Commun. Nanotechnol., no. Iceccn, pp. 227-231, Mar. 2013.

[12] C. Brunner and R. Leeb, "BCI Competition 2008 - Graz data set A," pp. 1-6, 2008.

[13] P. von Bunau, F. C. Meinecke, F. Kiraly, and K.-R. Muller, "Finding stationary subspaces in multivariate time series.," Phys. Rev. Lett., vol. 103, 2009.

[14] W. Samek, K.-R. Muller, M. Kawanabe, and C. Vidaurre, "Brain-computer interfacing in discriminative and stationary subspaces.," Conf. Proc. ... Annu. Int. Conf. IEEE Eng. Med. Biol. Soc. IEEE Eng. Med. Biol. Soc. Annu. Conf., Jan. 2012.

[15] "EPAPS Document No. E-PRLTAO-103-014948." [Online]. Available: http://www.aip.org/pubservs/epaps.html. 
Bulletin de la Société Royale des Sciences de Liège, Vol. 85, 2016, p. 1204 - 1210

[16] H. Rastgoo and S. Azadi, "Improving Decoding of the Mental Activities in BCI Systems using Overlapping FBCSP,” vol. 75, no. February, pp. 95-99, 2016.

[17] “No Title.” [Online]. Available: http://www.bbci.de/competition/iv/results/\#dataset2a. 